\title{
AMENABLE ACTIONS AND WEAK CONTAINMENT OF CERTAIN REPRESENTATIONS OF DISCRETE GROUPS
}

\author{
M. GABRIELLA KUHN
}

(Communicated by J. Marshall Ash)

\begin{abstract}
We consider a countable discrete group $\Gamma$ acting ergodically on a standard Borel space $S$ with quasi-invariant measure $\mu$. Let $\pi$ be a unitary representation of $\Gamma$ on $L^{2}(S, d \mu, \mathscr{H})$ "nicely" related with $S$. We prove that if $\Gamma$ acts amenably on $S$ then $\pi$ is weakly contained in the regular representation.
\end{abstract}

Let $\Gamma$ be a countable discrete group acting (on the right) on a standard Borel space $S$. Suppose that the action is ergodic with $\mu$ as a quasi-invariant probability measure on $S$. Let $P(s, g)$ be the Radon-Nikodym cocycle of the action, that is, $P(s, g)=\frac{d \mu(s g)}{d \mu(s)}$. Construct a representation of $\Gamma$ as follows:

(1) choose any Hilbert space $\mathscr{H}$,

(2) choose any (Borel) cocycle $A(s, g): S \times G \rightarrow \mathscr{U}(\mathscr{H})$ where $\mathscr{U}(\mathscr{H})$ denotes the group of unitary operators on $\mathscr{H}$,

(3) construct $\mathscr{H}_{\pi}=L^{2}(S, d \mu, \mathscr{H})$ with the usual inner product given by $\langle F, G\rangle=\int_{S}\langle F(s), G(s)\rangle_{\mathscr{X}} d \mu(s)$,

and finally define

$$
(\pi(g) F)(s)=P(s, g)^{\frac{1}{2}} A(s, g) F(s g) .
$$

Observe now that although the group is not amenable it turns out that $\pi$ is weakly contained in the regular representation for many natural constructions. In particular, this is true when

(a) $\Gamma$ is a free group and $S$ is its Martin boundary (see [K-S, Appendix]),

(b) $\Gamma$ is a hyperbolic group and $S$ is its boundary (see [A] and use the techniques of [K-S]), and

(c) $\Gamma$ is a lattice in a semisimple Lie group $G$ and $S=G / B$ is the maximal Furstenberg boundary of $G$ (see [Q-S, $\S 4]$ ).

Recall that a group is amenable if and only if every unitary representation of $\Gamma$ is weakly contained in $\infty \pi_{\text {reg }}$ (where $\pi_{\text {reg }}$ denotes the regular representation) [H] and [Z3, Proposition 7.3.6].

Received by the editors February 10, 1993.

1991 Mathematics Subject Classification. Primary 43A07, 22D10.

Key words and phrases. Amenable actions, weak containment of representations. 
Zimmer introduced a class of Borel spaces which make a nonamenable group $G$ "look like" an amenable one when acting on them. These spaces are called amenable $G$-spaces ( $G$ is said to act amenably on them).

To see that cases (a) and (c) deal with amenable actions of $\Gamma$ on $S$ recall the following

Proposition [Z3]. Let $G$ be a locally compact group, $H$ a closed subgroup, and $\Gamma$ a lattice in $G$. Then $\Gamma$ acts amenably on $G / H$ if and only if $H$ is amenable.

Case (c) follows since minimal parabolic subgroups of semisimple Lie groups are amenable. For case (a) the reader should think of the free group acting on a homogeneous tree as a discrete cocompact subgroup of the full automorphism group $G$ of the tree. The Martin boundary of $\Gamma$ is the set of all "ends" of the tree, and it was proved by Nebbia [N] that the stabilizer (in $G$ ) of an end is amenable. Case (b) was proved by Adams in [A].

In this paper we shall prove the following

Theorem. Suppose that a countable discrete group $\Gamma$ acts ergodically on $(S, \mu)$ with quasi-invariant measure $\mu$. Suppose that the action is amenable. Then every representation $\pi$ constructed as above is weakly contained in $\pi_{\mathrm{reg}}$.

We also believe that the converse is true; namely, we believe that if for every Hilbert space $\mathscr{H}$ the above-constructed representation $\left(L^{2}(S, d \mu, \mathscr{H}), \pi\right)$ is weakly contained in $\pi_{\text {reg }}$ then the action is amenable.

Proof of the result. Denote by $\pi_{\text {reg }}$ the right regular representation of $\Gamma$. Construct $\pi_{\text {reg }} \otimes \pi$ acting on $\ell^{2}(\Gamma) \otimes \mathscr{H}_{\pi}$. Recall that $U: \ell^{2}(\Gamma) \otimes \mathscr{H}_{\pi} \rightarrow \ell^{2}\left(\Gamma, \mathscr{H}_{\pi}\right)$ given by

$$
(U f)(x)=\pi(x) f(x)
$$

intertwines $\pi_{\text {reg }} \otimes \pi$ to $\pi_{\text {reg }} \otimes I$, where $I$ denotes the trivial representation. Thus $\pi_{\text {reg }} \otimes \pi$ is equivalent to a direct sum of copies of $\pi_{\text {reg }}$, and it is sufficent to prove that $\pi$ is weakly contained in $\pi_{\text {reg }} \otimes \pi$.

Fix any finite subset $E$ of $\Gamma$. Let $F \in L^{2}(S, d \mu, \mathscr{H})$. Inside $\ell^{2}(\Gamma) \otimes$ $L^{2}(S, d \mu, \mathscr{H})$ we shall construct a sequence of functions $\left(f_{n}\right)_{n=0}^{\infty}$ such that $\left\langle\pi_{\text {reg }} \otimes \pi(x) f_{n}, f_{n}\right\rangle$ approaches $\langle\pi(x) F, F\rangle$ for $x$ in $E$.

Identify $\ell^{2}(\Gamma) \otimes L^{2}(S, d \mu, \mathscr{H})$ with $L^{2}\left(S, d \mu, \ell^{2}(\Gamma) \otimes \mathscr{H}\right)$. Set $f_{n}(s)=$ $\phi_{n}(s) \otimes F(s)$ where the $\phi_{n}$ are elements of

$$
\ell^{2}(\Gamma) \otimes L^{2}(S, d \mu)=L^{2}\left(S, d \mu, \ell^{2}(\Gamma)\right) .
$$

Compute

$$
\begin{aligned}
\left\langle\pi_{\mathrm{reg}}\right. & \left.\otimes \pi(g) f_{n}, f_{n}\right\rangle \\
& =\int_{S} P(s, g)^{\frac{1}{2}}\left\langle\pi_{\mathrm{reg}}(g) \phi_{n}(s g), \phi_{n}(s)\right\rangle_{\ell^{2}(\Gamma)}\langle A(s, g) F(s g), F(s)\rangle_{\mathscr{X}} d \mu(s)
\end{aligned}
$$

and

$$
\langle\pi(g) F, F\rangle=\int_{S} P(s, g)^{\frac{1}{2}}\langle A(s, g) F(s g), F(s)\rangle_{\mathscr{C}} d \mu(s) .
$$

In order to prove our result we need a sequence of functions $\phi_{n}(s, x) \in$ $L^{2}\left(S, d \mu, \ell^{2}(\Gamma)\right)$ such that $\sum_{x \in \Gamma} \phi_{n}(s g, x g) \phi_{n}(s, x)$ is weakly converging, in $L^{2}(S, d \mu)$, to the function identically one on $S$. 
For amenable groups the existence of such a sequence is related to existence of an invariant mean in $\left(L^{\infty}(G)\right)^{*}$.

An analogue of the invariant mean property was proved for discrete groups by Zimmer in $[\mathrm{Z} 1, \mathrm{Z2}]$ in the case of an amenable action. The reader may take the following as a definition of an amenable action:

Proposition 1 (Zimmer). Suppose that $\Gamma$ is a countable discrete group acting ergodically on $(S, \mu)$. Then $S$ is an amenable $\Gamma$-space if and only if there is a norm one linear map $\sigma: L^{\infty}(S \times \Gamma) \rightarrow L^{\infty}(S)$ such that:

(1) $\sigma(\mathbf{1})=\mathbf{1}, f \geq 0$ implies $\sigma(f) \geq 0$;

(2) if $A \subseteq S$ is measurable, $\sigma\left(f \cdot \chi_{p^{-1} A}\right)=\sigma(f) \chi_{A}$ where $p: S \times \Gamma \rightarrow S$ is the projection; and

(3) $\sigma(f \cdot g)=\sigma(f) \cdot g$, where $f \cdot g(s, x)=f(s g, x g)$ and $\phi \cdot g(s)=\phi(s g)$.

In [Z1] it is shown how to construct $\sigma$ once we know that the action of $\Gamma$ on $S$ is amenable. From these we may deduce other properties of $\sigma$ which will be needed. For the reader's convenience we briefly recall the construction. Let $\tilde{U}_{g}: L^{2}(S \times \Gamma) \rightarrow L^{2}(S \times \Gamma)$ be defined by

$$
\left(\tilde{U}_{g} f\right)(s, x)=f(s g, x g) P(s, g)^{\frac{1}{2}} ; .
$$

For $f \in L^{\infty}(S)$ define a multiplication operator $M_{f}$ on $L^{2}(S \times \Gamma)$ by letting

$$
\left(M_{f} h\right)(s, x)=f(s) h(s, x) .
$$

Let $R$ denote the von Neumann algebra generated by $\left\{\tilde{U}_{g}, M_{f}\right\}$ in $\mathscr{B}\left(L^{2}(S \times \Gamma)\right)$. It was proved in [Z2] that if $\Gamma$ acts amenably on $S$ then there is a norm one projection and hence a conditional expectation $P: \mathscr{B}\left(L^{2}(S \times \Gamma)\right) \rightarrow$ $R$.

Consider now the following decomposition for $L^{2}(S \times \Gamma)$. For $f \in L^{2}(S \times \Gamma)$ let $f_{s}(x)=f(s x, x) P(s, x)^{\frac{1}{2}}$. Then $f \rightarrow \int_{S}^{\oplus} f_{s} d \mu$ is a unitary isomorphism of $L^{2}(S \times \Gamma) \simeq \int_{S}^{\oplus} \ell^{2}(\Gamma) d \mu$. Further every element of $R$ is decomposable with respect to this direct integral decomposition. (Namely, $\tilde{U}_{g}$ corresponds to $\int_{S}^{\oplus} \pi_{\text {reg }}(g)$ and $M_{f}$ to $\int_{S}^{\oplus} f^{s}$ where $\left(f^{s} h_{s}\right)(x)=f(s x) h_{s}(x)$.) For every $f \in L^{\infty}(S \times \Gamma)$ we have the multiplication operator $M_{f} \in \mathscr{B}\left(L^{2}(S \times \Gamma)\right)$. Now $P\left(M_{f}\right)$ is decomposable; hence, we can write $P\left(M_{f}\right)=\int_{S}^{\oplus} T_{s}^{f} d \mu$. Our map $\sigma$ is now given by

$$
(\sigma f)(s)=\left\langle T_{s}^{f}\left(\delta_{e}\right), \delta_{e}\right\rangle_{\ell^{2}(\Gamma)},
$$

where $\delta_{e}$ denotes the characteristic function of the identity $e$.

In particular, if $F$ is an element of $L^{\infty}(S)$ and we let $F(s, g)=F(s)$ for every $g \in \Gamma$ then

$$
\text { (i) }(\sigma F)(s)=F(s) \text {; }
$$

furthermore, for every $h \in L^{\infty}(S \times \Gamma)$

$$
\text { (ii) }(\sigma h F)(s)=(\sigma h)(s) F(s) \text {, }
$$

since $P\left(M_{h} M_{F}\right)=P\left(M_{h}\right) M_{F}$ because $F$ is an element of $R$.

In order to use our function $\sigma$ we need the Radon-Nikodym derivative $P(s, g)^{\frac{1}{2}}$ to be a bounded function of $s$ for any fixed $g$. This, in general, may or may not be true. However, we shall prove that it is always possible to find a measure $\nu$ equivalent to $\mu$, for which this is true. 
Lemma 1. Let $\Gamma$ be a discrete group. There exists a length function $x \rightarrow\|x\|$ such that the cardinality of the sets $\{\|x\| \leq N\}$ is less than or equal to $3^{N}$. Given such a length function choose $K>3$ and set

$$
\nu(E)=\sum_{x \in \Gamma} K^{-\|x\|} \mu_{x}(E) \quad \text { where } \mu_{x}(E)=\mu(E x)
$$

for every measurable $E$. Then $\nu$ is equivalent to $\mu$ and the Radon-Nikodym derivative $\frac{d \nu(s g)}{d \nu(s)}$ is less than or equal to $K^{\|g\|}$.

Proof. Choose any ordering $\left\{x_{n}\right\}_{n=0}^{\infty}$ for the elements of $\Gamma$ satisfying $x_{0}=e$. Set

$$
\|x\|=\inf \left\{\sum_{j=1}^{J} n_{j}: x=x_{n_{1}}^{ \pm} x_{n_{2}}^{ \pm} \cdots x_{n_{j}}^{ \pm}\right\} .
$$

It is immediate that $\|\cdot\|$ is a length function. Set $\Phi(N)=\sharp\{x:\|x\|=N\}$. We observe that the number of strictly positive solutions $\left(n_{1}, n_{2}, \ldots, n_{k}\right)$ of the equation

$$
n_{1}+n_{2}+\cdots+n_{k}=N
$$

is $\left(\begin{array}{c}N-1 \\ k-1\end{array}\right)$. Hence

$$
\Phi(N) \leq \sum_{k=1}^{N}\left(\begin{array}{l}
N-1 \\
k-1
\end{array}\right) 2^{k}=2 \cdot 3^{N-1}
$$

Finally

$$
\sharp\{x:\|x\| \leq N\} \leq 1+2+2 \cdot 3+\cdots+2 \cdot 3^{N-1}=3^{N} .
$$

It is obvious from the definition of $\nu$ and from the quasi-invariance of $\mu$ that $\nu$ and $\mu$ are equivalent measures. Let $E$ be a measurable set. Compute

$$
\begin{aligned}
\nu(E g) & =\sum_{x \in \Gamma} K^{-\|x\|} \mu(E g x)=\sum_{x \in \Gamma} K^{-\|x\|-\|g\|+\|g\|} \mu(E g x) \\
& \leq K^{\|g\|} \sum_{x \in \Gamma} K^{-\|g x\|} \mu(E g x)=K^{\|g\|} \nu(E) .
\end{aligned}
$$

This completes the proof.

From this point on we shall assume that the Radon-Nikodym derivative $P(s, g)$ is a bounded function of $s$ for any given $g$.

Proof of the theorem. Let

$$
P=\left\{\phi \in L^{1}(S \times \Gamma) \mid \phi(s, x) \geq 0 \text { and } \int_{S} \sum_{x \in \Gamma} \phi(s, x) d \mu(s)=1\right\} .
$$

First of all observe that $P$ is weak- ${ }^{*}$ dense in the unit ball of $\left(L^{\infty}(S \times \Gamma)\right)^{*}$. Construct a linear functional $\sigma^{*}$ on $L^{\infty}(S \times \Gamma)$ by letting

$$
\sigma^{*}(f)=\int_{S}(\sigma f)(s) d \mu(s) .
$$

Recall that $(h \cdot g)(s, x)=h(s g, x g)$ and $(\psi \cdot) g(s)=\psi(s g)$. 
Choose $h$ in $L^{\infty}(S \times \Gamma), \epsilon$ positive, and $g$ in a finite set $E$ of $\Gamma$. First of all we shall show that it is possible to find $\phi \in L^{1}(S \times \Gamma)$ such that

$$
\left|\int_{S}\left(\sum_{x \in \Gamma}(\phi(s g, x g)-\phi(s, x)) h(s, x)\right) d \mu(s)\right|<\epsilon \quad \text { for every } g \text { in } E \text {. }
$$

In fact, by property (c) of Proposition 1, we have

$$
\begin{aligned}
\int_{S}(\sigma h)(s) d \mu(s) & =\int_{S}\left(\sigma h \cdot g^{-1}\right)(s) P\left(s, g^{-1}\right) d \mu(s) \quad \text { (because of (ii)) } \\
& =\int_{S}\left(\sigma h \cdot g^{-1} P\left(s, g^{-1}\right)\right)(s) d \mu(s) .
\end{aligned}
$$

Hence it is possible to choose $\phi \in L^{1}(S \times \Gamma)$ so that

$$
\left|\int(\sigma h)(s)-\left(\sum_{x \in \Gamma} \phi(s, x) h(s, x)\right) d \mu(s)\right|<\epsilon
$$

and

$$
\left|\int_{S}\left(\sigma h \cdot g^{-1} P\left(s, g^{-1}\right)\right)(s)-\left(\sum_{x \in \Gamma} \phi(s, x) h\left(s g^{-1}, x g^{-1}\right) P\left(s, g^{-1}\right)\right) d \mu(s)\right|<\epsilon .
$$

But

$$
\begin{array}{r}
\int_{S}\left(\sum_{x \in \Gamma} \phi(s, x) h\left(s g^{-1}, x g^{-1}\right) P\left(s, g^{-1}\right)\right) d \mu(s) \\
=\int_{S} P\left(s, g^{-1}\right) \sum_{x \in \Gamma} \phi(s, x g) h\left(s g^{-1}, x\right) d \mu(s)
\end{array}
$$

and letting $s g^{-1}=s^{\prime}$

$$
=\int_{S} \sum_{x \in \Gamma} \phi(s g, x g) h(s, x) d \mu(s) .
$$

Subtracting (1.3) from (1.2) we get (1.1)

Moreover, since $\sigma(1)=1$, we may also require that for any given $h^{\prime} \in L^{\infty}(S)$

$$
\left|\int_{S} h^{\prime}(s)\left(1-\sum_{x \in \Gamma} \phi(s, x)\right) d \mu(s)\right|<\epsilon .
$$

We shall use now Namioka's argument to pass from the weak to the strong closure. Let $F=L^{1}(S) \times \prod_{g \in \Gamma} L^{1}(S \times \Gamma)=F_{0} \times \prod_{g \in \Gamma} F_{g}$ where $L^{1}(S)$ and each copy of $L^{1}(S \times \Gamma)$ has the norm topology. The weak topology on $F$ is the product topology for the weak topology on $L^{1}(S)$ and on $L^{1}(S \times \Gamma)$. Define a linear map $T: P \rightarrow F$ by letting

$$
(T(\phi))_{0}=\sum_{x \in \Gamma} \phi(s, x) \quad \text { and } \quad(T(\phi))_{g}=\phi(s g, x g)-\phi(s, x) .
$$


Then (1.1) and (1.4) say that the point $(1,0, \ldots, 0 \ldots)$ is in the weak closure of $T(P)$, and since $T(P)$ is convex, it follows that the same point is in the strong closure of $T(P)$. Hence we can find a net of elements $\psi_{j} \in P$ such that

$$
\sum_{x \in \Gamma} \psi_{j}(s, x) \rightarrow 1 \text { and } \psi_{j}(s g, x g)-\psi_{j}(s, x) \rightarrow 0
$$

strongly, respectively, in $L^{1}(S)$ and in $L^{1}(S \times \Gamma)$.

Finally let $\phi_{j}(s, x)=\sqrt{\psi_{j}(s, x)}$. By standard arguments

$$
\left\|\phi_{j} \cdot g-\phi_{j}\right\|_{L^{2}(S \times \Gamma)} \leq\left\|\psi_{j} \cdot-\psi_{j}\right\|_{L^{1}(S \times \Gamma)}^{\frac{1}{2}} .
$$

Choose any $F$ in $L^{2}(S, d \mu, \mathscr{H})$. Set $f_{n}(s, x)=\phi_{n}(s, x) \otimes F(s)$. Let

$$
G(s, g)=P(s, g)^{\frac{1}{2}}\langle A(s, g) F(s g), F(s)\rangle_{\mathscr{H}} \text {. }
$$

Since the set of functions $F: S \rightarrow \mathscr{H}$ such that $\langle(\pi(g) F)(s), F(s)\rangle$ is a bounded function of $s$ is dense in $L^{2}(S, d \mu, \mathscr{H})$, we may assume that $G(s, g)$ is a bounded function of $s$ for any given $g \in \Gamma$. We may also assume that $G(s, g) \geq 0$. Observe that $\left\|\sqrt{G(s, g)}\left(\phi_{n} \cdot g-\phi_{n}\right)\right\|_{L^{2}(S \times \Gamma)} \rightarrow 0$. We have

$$
\begin{aligned}
\left\|\sqrt{G(s, g)}\left(\phi_{n} \cdot g-\phi_{n}\right)\right\|_{L^{2}(S \times \Gamma)} & \\
= & \int_{S} G(s, g) \sum_{x \in \Gamma} \psi_{n}(s g, x g) d \mu(s)+\int_{S} G(s, g) \sum_{x \in \Gamma} \psi_{n}(s, x) d \mu(s) \\
& -2 \int_{S} G(s, g) \sum_{x \in \Gamma}\left(\phi_{n}(s g, x g) \phi_{n}(s, x)\right) d \mu(s) .
\end{aligned}
$$

But $\sum_{x \in \Gamma} \psi_{n}(s, x)$ converges to 1 in $L^{1}(S)$, and so does $\sum_{x \in \Gamma} \psi_{n}(s g, x g)$ since $\left\|\psi_{n} \cdot g-\psi_{n}\right\|_{L^{1}(S \times \Gamma)} \rightarrow 0$. Hence,

$$
\int_{S} G(s, g) \sum_{x \in \Gamma}\left(\phi_{n}(s g, x g) \phi_{n}(s, x)\right) d \mu(s) \rightarrow \int_{S} G(s, g) d \mu(s) \text {. }
$$

\section{ACKNOWLEDGMENT}

We are happy to thank Tim Steger for suggesting the proof of Lemma 1. A special thanks to Robert Zimmer for helpful conversations and remarks and for providing us with $[\mathrm{Z} 1]$ and $[\mathrm{Z} 2]$.

\section{REFERENCES}

[A] S. Adams, Boundary amenability for hyperbolic groups and an application to smooth dynamic of simple groups, preprint.

[H] A. Hulanicki, Means and Folner conditions on locally compact groups, Studia Math. 27 (1966), 87-104.

[K-S] M. G. Kuhn and T. Steger, More irreducible boundary representations of free groups, preprint.

[N] C. Nebbia, Amenabilty and Kunze-Stein property for groups acting on a tree, Pacific J. Math. 135 (1988), 371-380.

[Q-S] J. C. Quigg and J. Spielberg, Regularity and hyporegularity in $C^{*}$-dynamical systems, Houston J. Math. 18 (1992), 139-152. 
[Z1] R. J. Zimmer, On the von Neumann algebra of an ergodic group action, Proc. Amer. Math. Soc. 66 (1977), 289-293.

[Z2] — Hyperfinite factors and amenable ergodic actions, Invent. Math. 41 (1977), 23-31.

[Z3] _ Ergodic theory and semisimple groups, Birkhäuser, Boston, MA, 1984.

Dipartimento di Matematica, Universita degli Studi di Milano, via C. Sadini, 5020133 Milano, Italy

E-mail address: kuhn@vmimat mat.unimi.it 